Meta

Journal des traducteurs

Translators' Journal

\title{
Les étapes de la fabrication des circuits intégrés : termes en contexte
}

\section{Jacques Lethuillier}

Volume 26, numéro 4, décembre 1981

URI : https://id.erudit.org/iderudit/003480ar

DOI : https://doi.org/10.7202/003480ar

Aller au sommaire du numéro

Éditeur(s)

Les Presses de l'Université de Montréal

ISSN

0026-0452 (imprimé)

1492-1421 (numérique)

Découvrir la revue

Citer cet article

Lethuillier, J. (1981). Les étapes de la fabrication des circuits intégrés : termes en contexte. Meta, 26(4), 359-369. https://doi.org/10.7202/003480ar d'utilisation que vous pouvez consulter en ligne.

https://apropos.erudit.org/fr/usagers/politique-dutilisation/ 


\section{ÉTUDES \\ TERMINOLOGIQUES \\ ET LINGUISTIQUES}

\section{LES ÉTAPES DE LA FABRICATION DES CIRCUITS INTÉGRÉS: TERMES EN CONTEXTE}

Les circuits intégrés monolithiques (monolithic integrated circuits) sont implantés sur un support fait d'un solide semiconducteur (semiconductor, semiconducting material), c'est-à-dire d'une substance dont le «comportement électrique» s'apparente tantôt à celui des conducteurs (conductors), tantôt à celui des isolants (insulators).

Les conducteurs sont des matériaux qui recèlent des porteurs de charge libres (mobile charge carrier), le plus souvent des électrons. Ces derniers peuvent recevoir une quantité infime d'énergie, sous l'effet d'un champ électrique, par exemple. A leur mouvement désordonné correspondant à l'agitation thermique se superpose alors un mouvement d'ensemble selon la direction du champ électrique : c'est le courant électrique.

Les isolants, eux, n'ont pas d'électrons libres. Tous les électrons, dans un isolant, participent à des liaisons (bond) au sein de la gigantesque molécule que forme chaque grain cristallin (crystallite, grain). Aucun courant n'apparaît donc lorsque l'on applique un champ électrique à un tel corps.

Les semiconducteurs, enfin, ont des propriétés qui les apparentent aux isolants, d'autres qui les font considérer comme des conducteurs. Au zéro absolu $\left(-273{ }^{\circ} \mathrm{K}\right)$, les semiconducteurs purs sont des isolants parfaits. Tous les électrons sont engagés dans des liaisons. Même les électrons périphériques (peripheral electron) restent liés à un noyau particulier. Mais à mesure que s'élève la température, des liaisons se brisent et libèrent des électrons périphériques ou électrons de valence (valence electron). Le corps devient partiellement conducteur.

Pour de tels corps, la conductivité électrique (electrical conductivity), qui mesure l'aptitude d'une substance à conduire l'électricité et s'exprime en mhos (mho), augmente de façon très marquée avec la température. C'est l'inverse de ce que l'on observe avec les conducteurs, dont la résistivité (resistivity) crôt lorsque la température s'élève, par suite de l'importance que prennent les phénomènes de collision entre les noyaux du cristal et les électrons.

On distingue deux grandes catégories de semiconducteurs: les semiconducteurs intrinsèques (intrinsic semiconductor) et les semiconducteurs extrin- 
sèques (extrinsic semiconductor, impurity semiconductor). C'est l'agitation thermique, donc l'accroissement de la température, qui fait apparaître des porteurs libres au sein des semiconducteurs de la première catégorie. Le germanium illustre de façon typique ce "comportement". Dans le cas des semiconducteurs extrinsèques, on incorpore des impuretés (impurity) au cristal pour obtenir le même résultat. L'action d'introduire des atomes étrangers dans certains corps ayant naturellement la structure d'isolant, en vue de les rendre conducteurs, s'appelle le dopage (doping).

$\mathrm{Au}$ silicium (silicon), par exemple, on ajoute, en quantité extrêmement faible, des atomes d'un élément ayant une valence (valence) de trois comme le bore (boron) ou de cinq comme le phosphore (phosphorus). Autrement dit, des atomes d'un élément dont le nombre d'électrons de valence est supérieur ou inférieur d'une unité à celui du silicium qui est de quatre. Selon que la valence de l'impureté est supérieure ou inférieure à celle du semiconducteur, il se crée une prédominance de porteurs libres négatifs ou positifs. C'est dire que la conduction se fait essentiellement soit par des électrons, soit par des lacunes électroniques ou trous. On parle alors de semiconducteurs de type $\mathbf{n}$ (n-type semiconductor) ou de semiconducteurs de type $\mathbf{p}$ (p-type semiconductor).

Les électroniciens ont su tirer un parti extraordinaire de ces semiconducteurs extrinsèques, dont on peut modifier localement les propriétés électriques, et en particulier la conductivité, en jouant sur la nature des impuretés introduites et leur concentration.

En microélectronique, c'est essentiellement grâce au dopage que des effets spéciaux sont obtenus pour la réalisation de composants actifs (active component, active element). Les jonctions (junction), par exemple, qui sont à la base de la fabrication des diodes solides (solid-state diode) et des transistors, résultent de la juxtaposition, au sein d'un même cristal, d'une zone semi-conductrice de type $n$ et d'une zone de type p. Même les composants passifs (passive component) interconnexions et isolements - peuvent être réalisés en faisant varier les concentrations d'impuretés.

Actuellement, le support choisi pour servir de substrat à un circuit intégré est le silicium. Ce support, qui possède une conductibilité de type donné, se présente sous la forme d'un monocristal (single crystal). De façon générale, un monocristal est assimilable à un réseau cristallin (crystal lattice) caractérisé par un motif élémentaire (unit cell), forme géométrique simple comme le cube ou le parallélépipède. À chacun des sommets (vertex) du cube ou du parallélépipède se trouve un atome ou un groupe d'atomes définis. Si l'on reproduit un tel motif élémentaire dans les trois dimensions, on obtient un monocristal.

À l'état naturel, cependant, la plupart des substances sont polycristallines (polycrystalline). Elles résultent de la juxtaposition de grains monocristallins (crystallite, grain) très petits, discernables au microscope seulement, qui sont orientés au hasard. Dans un bloc de matière donné, tous les grains ont le même motif, le même pas (lattice constant, lattice spacing), mais l'orientation de motifs change au passage d'une frontière (boundary) commune à deux grains voisins. 
La fabrication des monocristaux servant à réaliser les circuits intégrés monolithiques se fait souvent par croissance (growth) à partir d'un bain de silicium en fusion (melt) dans un creuset (crucible), selon la méthode de tirage de Czochraslski. Après adjonction de la quantité d'impuretés voulue, une amorce cristalline, appelée germe (seed) et de même nature que le cristal à tirer, est amenée avec précaution au contact de la surface liquide. Puis le germe est tiré au moyen d'une tige conique à son extrémité, que l'on remonte lentement tout en la faisant tourner et en abaissant la température du bain pour compenser l'apport de chaleur occasionné par la cristallisation progressive. Toute l'opération se déroule dans une atmosphère constituée par un gaz inerte, de façon à empêcher la contamination ou l'oxydation du silicium à l'état liquide. On obtient, par la mise en œuvre de cette méthode, des lingots ou barreaux cylindriques (cylinder) de silicium monocristallin dopé à la concentration voulue, qui fournissent le matériau de support des circuits intégrés.

Ces lingots ou barreaux sont ensuite découpés en tranches (to slice) par sciage. On soumet les lames ou tranches ${ }^{1}$ (slice, wafer) ainsi obtenues à des traitements mécaniques avant de leur faire subir une passivation (passivation) chimique. Par meulage (grinding), les deux faces sont égalisées ou aplanies (smooth$e d$ ), puis l'une d'entre elles est finement polie (highly polished).

À ce stade, il reste à protéger la surface de silicium, des deux côtés de la tranche, par oxydation : c'est la passivation. Un lot de tranches (batch) est disposé à l'intérieur d'une ampoule ou capsule de quartz (quartz boat), et le tout est introduit (to load) dans un four à oxydation (oxydation furnace). Les tranches de silicium sont chauffées en présence d'oxygène ou de vapeur d'eau, que l'on fait circuler à travers l'ampoule de quartz. Une fine pellicule de silice ou bioxyde de silicium, ayant l'aspect d'un verre transparent (clear glass), se forme alors en surface. Le film est dur et durable. Il adhère bien et constitue un bon isolant. Au cours des étapes ultérieures d'implantation proprement dite des composants, on s'en servira comme d'un masque. Grâce à lui, le dopage sélectif du silicium, c'est-à-dire le dopage d'une zone donnée à la concentration voulue, sera réalisable. Par ailleurs, quand le circuit sera terminé, la couche d'oxyde le protégera contre l'oxydation et l'humidité.

La passivation marque la fin de la préparation des tranches. À cette dernière étape préparatoire fait suite l'implantation des composants actifs et passifs, en couches superposées dans la masse ou à la surface du support semiconducteur, selon le plan (layout) du concepteur de circuit (circuit designer). Sur les tranches, qui ont trois ou quatre centimètres de diamètre et un dixième de millimètre d'épaisseur, quelque deux cents circuits identiques - occupant chacun un carré de deux à trois millimètres d'arête (two or three millimeters across) et comportant plusieurs milliers de composants élémentaires - seront fabriqués. Ce sont ces petits carrés, une fois découpés pour former des circuits distincts, que l'on appelle puces (chip) ou pastilles (die), ou bien parfois encore microplaquettes (chip).

1. Aussi : rondelle et plaquette (ces deux termes sont beaucoup plus rarement employés). 
Les circuits intégrés monolithiques sont des structures électroniques à niveaux multiples. Des couches successives de composants élémentaires sont d'abord implantées en profondeur dans le support semiconducteur, puis à la surface de ce dernier. Dans les deux cas des masques (mask) traduisent la définition topologique à un niveau donné. Ils permettent de pratiquer par photogravure (photoetching), au niveau de la pellicule d'oxyde protectrice, les ouvertures ou fenêtres (window) nécessaires pour l'implantation des composants. Sur chaque masque, le dessin des ouvertures à travers lesquelles seront introduites les impuretés pour créer des zones localisées de conductibilité définie, est reproduit autant de fois qu'il y a de circuits à réaliser sur la tranche de silicium. Le jeu complet des masques donne la définition du circuit dans l'espace, son architecture.

La photogravure se déroule en plusieurs étapes. On commence par enduire la pellicule d'oxyde d'une résine ou laque photosensible (light-sensitive resin, photoresist). Puis, à travers le masque définissant les ouvertures à pratiquer, la tranche est exposée à un rayonnement ultraviolet (ultraviolet radiation). Seules ne sont pas éclairées (illuminated) les zones de la résine disposées sous les régions opaques du masque, qui correspondent aux fenêtres à ouvrir. Sur le reste de la tranche, la résine, sous l'effet du rayonnement, se sera polymérisée (to polymerize). Le lavage au moyen d'un solvant organique comme le trichloréthylène ne permettra d'ôter que les zones non polymérisées, c'est-à-dire n'ayant pas été soumises au rayonnement. Ensuite, en plongeant la tranche dans un bain d'acide fluorhydrique (hydrofluoric acid), on fera s'y dissoudre l'oxyde de silicium au niveau des fenêtres.

Ainsi ouvrée, la pellicule protectrice de silicium fait fonction de masque. Le semiconducteur est à nu aux endroits où il le faut. On peut passer à l'implantation physique des composants, par dopage ou dépôt sélectifs.

Pour le dopage, auquel on a recours afin de réaliser des couches de composants dans la masse du semiconducteur, deux techniques existent : la diffusion thermique (thermal diffusion) et l'implantation ionique (ion implantation). Mais seule la première des deux techniques est couramment employée.

Dans la diffusion thermique, le support est chauffé en présence des impuretés, à l'état de vapeur, qu'il faut introduire. Celles-ci pénètrent plus ou moins, sous l'effet de la chaleur, par diffusion. Le plus souvent, ce procédé se déroule en deux étapes. Au cours d'une première phase, dite de prédiffusion (predeposit step), le semiconducteur préalablement travaillé est porté à une température relativement basse dans un premier four où il est en contact avec la vapeur dopante (doping vapor). Comme la température est assez faible, les impuretés restent en surface. La durée de l'opération détermine la quantité d'impuretés introduites, donc la concentration. À la prédiffusion fait suite la phase de redistribution (drive-in diffusion step) qui s'effectue à température plus élevée, dans un deuxième four, en l'absence d'impuretés. Au cours de cette phase, dont la durée conditionne la profondeur de la pénétration (diffusion depth), la concentration d'impuretés en surface diminue à mesure que les impuretés s'enfoncent à l'intérieur du semiconducteur. 
La deuxième technique de dopage sélectif des semiconducteurs, l'implantation ionique, bien qu'à ses débuts, n'en est pas moins très prometteuse. Elle consiste à bombarder la tranche semiconductrice, après masquage, par un faisceau d'ions d'impureté choisie. Pour le bombardement (firing), on utilise un accélérateur de particules (particle accelerator). La profondeur à laquelle s'immobilise l'ion projeté (fired) dépend de sa vitesse, de sa taille par rapport à la distance interatomique du réseau et de l'orientation de ce dernier. De ce fait, la profondeur de pénétration est définissable avec une grande précision. Par ailleurs, il est possible de contrôler rigoureusement la concentration d'impuretés en mesurant la charge des ions.

Ainsi, l'implantation ionique conduit à des dopages beaucoup mieux définis que la diffusion. Elle présente cependant un inconvénient: les ions, en raison de leur grande énergie, bouleversent (to upset) l'organisation du réseau cristallin et en altèrent (to spoil) les propriétés électriques. On procède donc ordinairement au recuit (annealing) du silicium après l'implantation, de façon à redonner (to restore) au réseau sa configuration initiale. Jusqu'à présent, c'est le recuit par chauffage superficiel au moyen d'un faisceau laser qui donne les meilleurs résultats.

En résumé, le principal avantage de l'implantation ionique est sa précision. Néanmoins, pour tirer pleinement parti des possibilités qu'offre cette technique et réaliser des dispositifs élémentaires ayant des dimensions de l'ordre du micromètre, il faudrait employer des faisceaux d'électrons ou de rayons $\mathrm{X}$ pour transcrire, sur la couche protectrice recouvrant la tranche, le dessin des ouvertures à pratiquer. Ces rayonnements ayant des longueurs d'onde beaucoup plus faibles que le rayonnement ultraviolet, le phénomène de diffraction n'intervient pas pour limiter la finesse des éléments les plus petits. Il est vraisemblable d'ailleurs qu'un jour on ira encore beaucoup plus loin avec l'implantation ionique et que l'on parviendra à éliminer l'étape intermédiaire du masquage. Les mouvements du faisceau d'ions pourraient en effet être commandés par ordinateur. Grâce à un programme, on ferait décrire au faisceau un tracé prédéterminé et il modifierait directement les propriétés des zones correspondant aux éléments de circuits à implanter dans la masse du semiconducteur.

Les dopages sélectifs par diffusion thermique ou implantation ionique, dans la fabrication d'un circuit intégré typique, sont moins nombreux que les dépôts de couches minces (thin film deposition) qui servent à réaliser les couches de composants, le plus souvent passifs, couronnant la structure microélectronique. Parmi les procédés les plus couramment mis en œuvre à cette fin, on peut citer: l'évaporation sous vide (vacuum deposition, vacuum evaporation); la pulvérisation cathodique (cathodic sputtering); et le dépôt par voie gazeuse (chemical vapour deposition).

L'évaporation sous vide met en jeu une cloche (glass bell jar) où l'on maintient, grâce à une pompe à vide (vacuum pump), un vide assez poussé. Au bas de la cloche, dans un creuset (crucible) chauffé par le passage d'un courant électrique, se trouve le métal à déposer. À la partie supérieure, une nacelle (planetary) porte la tranche sur laquelle doivent être réalisés les composants en couche mince dont la forme est définie par les ouvertures d'un cache. Le cache 


\section{FABRICATION DES CIRGUITS INTEGRES}

Préparation du support

Préparation des plans

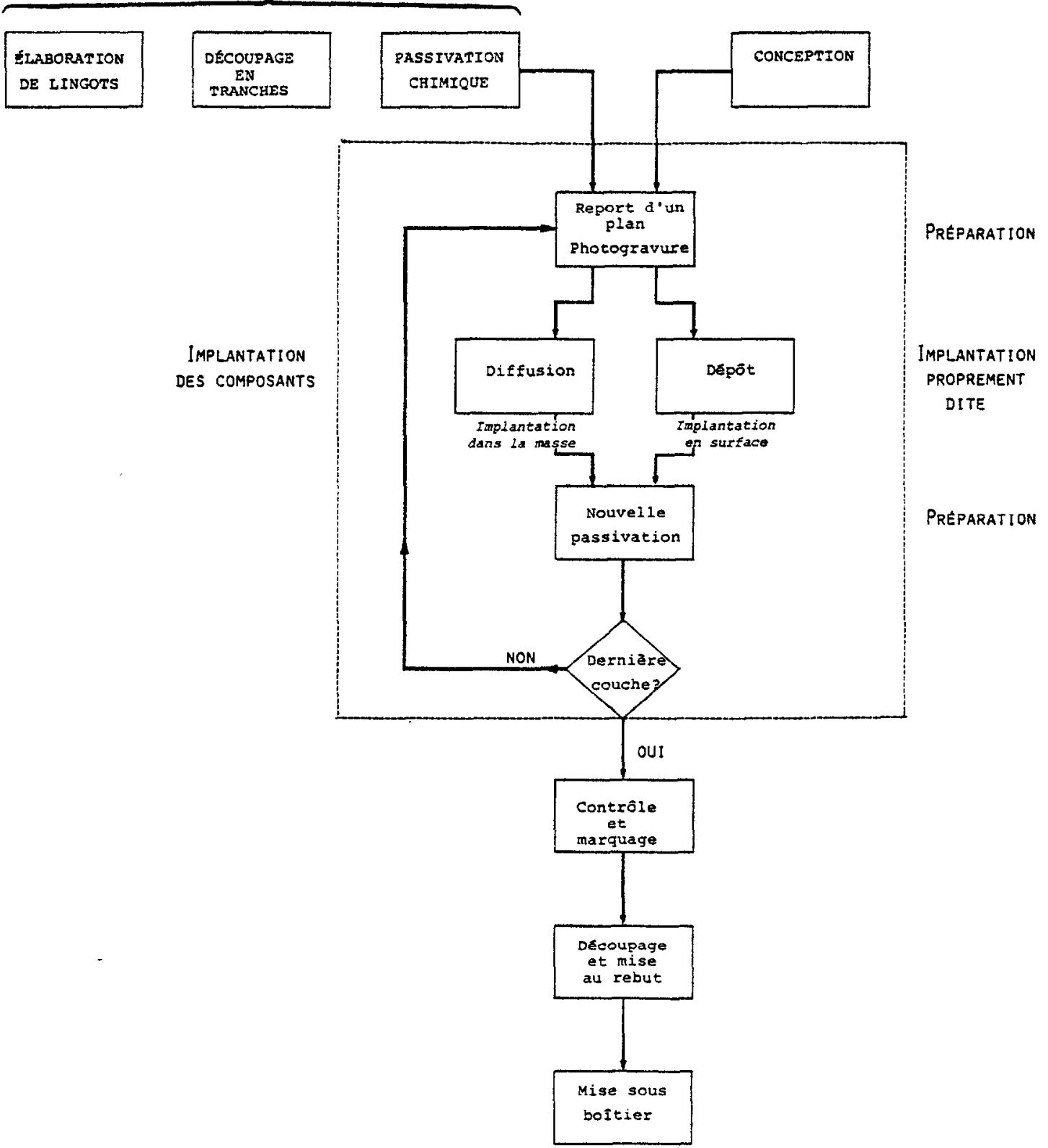


s'interpose entre le creuset et la nacelle. Il suffit de porter le métal dans le creuset au point de fusion pour que s'en échappent, par évaporation, les atomes de surface. Ces derniers se déplacent en droite ligne et recouvrent le circuit selon le tracé que définit le cache.

Pour des corps réfractaires (heat resistant) comme le tungstène (tungsten) ou le titane (titanium), l'évaporation est impossible ou très difficile. On recourt alors à la pulvérisation cathodique. Le principe en est simple. Il repose sur l'utilisation d'un tube à décharge gazeuse (gas discharge tube) dont la cathode est faite du matériau réfractaire à pulvériser. Le circuit sur lequel doit se faire le dépôt d'une couche mince est placé à proximité de l'anode, derrière un cache. Par suite de la décharge gazeuse, les ions du gaz résiduel bombardent la cathode et en éjectent les atomes qui diffusent à travers le plasma gazeux et se déposent sur la surface de la tranche semiconductrice.

À l'implantation de la dernière couche de composants fait suite un contrôle des circuits, exécuté automatiquement, au cours duquel les circuits défectueux sont marqués. Puis les tranches sont rainurées (to scribe) et brisées selon les lignes de rainurage. On obtient alors les circuits sous la forme de microplaquettes séparées. Les circuits marqués sont mis au rebut (to be discarded), tandis que les bons circuits sont encapsulés (encapsulated, capped), pour être ensuite mis sous boîtier (to package).

\section{LEXIQUE ANGLAIS-FRANÇAIS}

Active component - composant électronique actif, composant actif. Unité fonctionnelle élémentaire entrant dans la constitution des circuits électroniques. Un composant actif, de façon générale, sert à produire, à amplifier ou à transformer des signaux. Il est en communication avec au moins deux sources d'énergie, dont l'une sert à moduler le flux en provenance des autres.

Batch - lot (de tranches).

Bond - liaison. Lien entre deux atomes d'un «édifice» atomique - molécule de dimensions parfaitement définies ou monocristal formant une molécule géante aux dimensions indéfinies.

Boron - bore. Élément chimique (métallö̈de) trivalent que l'on utilise pour le dopage des semiconducteurs. En incorporant du bore au silicium, par exemple, on obtient un semiconducteur extrinsèque de type $p$, c'est-à-dire que l'on fait apparaitre dans le silicium des porteurs de charge libres qui sont des trous (lacunes électroniques).

Boundary - frontière. Limite entre deux grains d'une substance polycristalline.

Cap (to) - Voir to encapsulate.

Cathodic sputtering - pulvérisation cathodique. Procédé utilisé en micro-électronique pour déposer des métaux réfractaires à la surface d'un support semiconducteur. Pour les autres métaux, il est beaucoup moins rapide que l'évaporation sous vide.

Chip - puce, microplaquette. Chacune des petites surfaces élémentaires d'une tranche de silicium où un circuit intégré est implanté par mise en œuvre d'une suite d'opérations complexes. Les opérations successives sont exécutées simultanément pour toutes les petites surfaces élémentaires qui, à la fin du processus, sont séparées les unes des autres et constituent alors autant de circuits individuels.

Come to a rest (to) - s'immobiliser (pour un ion projeté à l'aide d'un accélérateur de particules, dans la technique de l'implantation ionique).

Conductivity - Voir electrical conductivity.

Conductor - conducteur. Substance constituant une réserve d'électrons libres aux températures ordinaires. Un très faible apport d'énergie peut y faire apparaître un courant. 
Crystal grain - grain cristallin, grain. Chacun des monocristaux dont se trouve composée une substance polycristalline. Au sein d'une telle substance, les monocristaux sont orientés au hasard et ils sont séparés par des frontières.

Crystal lattice - réseau cristallin. Représentation caractérisant la distribution régulière des atomes ou des molécules à l'intérieur d'un monocristal. Elle traduit la périodicité tridimensionnelle de ce dernier.

Crystallite - Voir crystal grain.

Die - pastille (terme souvent utilisé pour décrire ce qu'est une puce ou microplaquette).

Diffusion depth - profondeur de pénétration. Distance moyenne que parcourent les atomes d'impuretés introduites par diffusion thermique dans la masse du support où l'on veut créer localement une zone de conductivité électrique déterminée.

Discard (to) - mettre au rebut.

Doping - dopage. Action d'introduire dans la masse d'un semiconducteur des atomes d'une impureté choisie, en vue de lui donner - localement ou globalement, selon l'étape considérée - une conductivité de valeur déterminée.

Drive-in diffusion step - redistribution. Deuxième étape d'un dopage par diffusion thermique, qui se déroule dans un four comme la prédiffusion, mais en l'absence de vapeur dopante. Son rôle est de faire pénétrer les seules impuretés déjà incorporées au cours de l'étape antérieure (la prédiffusion).

Electrical conductivity - conductivité électrique. Aptitude d'un corps à se laisser traverser par un courant sous l'effet d'une tension électrique. C'est l'inverse de la résistivité.

Encapsulate (to) - encapsuler. Recouvrir une puce d'une résine protectrice, une fois le circuit réalisé et le contrôle de qualité effectué.

Extrinsic semiconductor - semiconducteur extrinsèque, semiconducteur dopé. Substance en principe isolante, que l'on rend faiblement conductrice aux températures ordinaires en lui incorporant, par dopage, une très faible quantité d'une impureté donnée.

Firing - bombardement. Action de projeter des particules (ions ou autres) sur une cible (cathode dans la pulvérisation cathodique et substrat semiconducteur dans l'implantation ionique).

Gas discharge tube - tube à décharge gazeuse. Tube électronique du type diode, contenant un gaz sous faible pression et que l'on utilise pour mettre en œuvre la pulvérisation cathodique. La cathode est faite du métal à déposer, et le substrat semiconducteur à la surface duquel on veut réaliser le dépôt d'une couche mince selon un tracé donné joue le rôle d'anode.

Glass bell jar - cloche.

Growth - croissance (d'un cristal). Pour un cristal, fait de se développer à partir d'un germe.

Heat resistant - réfractaire. Se dit des substances qui résistent à la chaleur et ne fondent qu'à des températures très élevées. Le dépôt de telles substances en couches minces sur des substrats semiconducteurs de circuit intégré, en vue de réaliser des composants passifs et notamment des résistances, exige que l'on ait recours à la pulvérisation cathodique, l'évaporation sous vide n'étant pas possible.

High vacuum - vide poussé.

Hole - trou. Lacune électronique en un site cristallin. Un trou peut être comblé par un électron provenant d'un autre site. On considère alors que le trou s'est déplacé. Un tel trou constitue un porteur de charge libre, de signe positif.

Hydrofuoric acid - acide fluorhydrique. Acide utilisé comme mordant au cours des opérations de photogravure mises en œuvre pour ouvrir des fenêtres dans la pellicule protectrice revêtant le support semiconducteur d'un circuit intégré.

Illuminate (to) - éclairer.

Impurity - impureté. Atome d'un élément trivalent ou pentavalent utilisé pour le dopage des semiconducteurs extrinsèques.

Impurity semiconductor - Voir extrinsic semiconductor.

Insulator - isolant. Substance au sein de laquelle tous les électrons des atomes sont fortement liés aux noyaux de ces derniers. Pour rendre libres les électrons les moins liés, c'est-à-dire ceux des couches périphériques, il faut leur apporter une énergie considérable. De telles substances ne laissent donc pas facilement passer un courant sous l'effet de l'application d'un champ électrique.

Intrinsic semiconductor - semiconducteur intrinsèque. Substance se comportant comme un isolant aux basses températures mais qui, à partir d'une certaine température, devient conductrice de l'électricité. Contrairement aux corps conducteurs ordinaires comme les métaux, la résistivité électrique de telles substances diminue lorsque la température augmente. 
Ion implantation - implantation ionique. Dopage d'un support semiconducteur par bombardement au moyen d'un faisceau d'ions de l'impureté choisie que produit un accélérateur de particules. Le support de circuit intégré, protégé par son masque, constitue la cible.

Junction - jonction. De façon générale, région de transition entre deux substances ayant des propriétés électriques (type de conductibilité et conductivité) différentes. Plus spécifiquement, dans un semiconducteur monocristallin, région de transition entre une zone de type $\mathrm{p}$ et une zone de type $\mathrm{n}$. La plupart des composants actifs à semiconducteurs (diode solide ou transistor, par exemple) comportent une ou plusieurs jonctions.

Lattice - Voir crystal lattice.

Lattice constant - pas (d'un réseau). Caractéristique d'un réseau correspondant à la distance séparant ses plans réticulaires.

Lattice spacing - Voir lattice constant.

Light-sensitive resin - Voir photoresist.

Mask - masque. Négatif obtenu par réduction photographique du dessin à grande échelle représentant une couche de composants d'un circuit intégré. Le négatif sert à reporter, par mise en cuvre de la technique de photogravure, le plan d'un niveau du circuit à réaliser sur la couche de passivation (oxyde de silicium).

Melt - bain (de silicium en fusion).

Mho - mho. Unité de mesure de la conductivité. C'est une anagramme de «ohm».

Mobile charge carrier - porteur de charge libre, porteur libre. Particule chargée électriquement (ou absence de particule), non liée à un site atomique en particulier et pouvant se déplacer librement à l'intérieur du réseau cristallin.

Monolithic integrated circuit - circuit intégré monolithique. Circuit fabriqué à partir d'un substrat semiconducteur (en silicium le plus souvent), dans lequel ou sur lequel sont réalisés, par implantation dans la masse ou dépôt en surface, aussi bien les composants actifs (diodes, transistors, etc.) que les composants passifs (condensateurs, résistances, interconnexions, etc.).

$N$-type semiconducteur - semiconducteur de type $\mathbf{n}$. Semiconducteur extrinsèque où la densité d'électrons de conduction dépasse celle des trous.

Outer electron - électron périphérique.

Oxydation furnace - four à oxydation. Dans la fabrication des circuits intégrés, four utilisé pour la passivation des supports semiconducteurs.

Package (to) - mettre sous boîtier.

Particle accelerator - accélérateur de particules. Appareil utilisé pour l'implantation ionique. Il comporte à son entrée une source de particules chargées et est conçu pour apporter aux particules du faisceau ainsi produit une grande quantité d'énergie, de façon à accroître considérablement leur vitesse.

Passive component - composant passif. Composant de circuit électronique comme les résistances, les condensateurs ou les inductances, ne recevant de l'énergie que d'une seule source et ayant un effet direct sur le flux d'énergie qui les traverse.

Passivation - passivation. Passage au four d'un support (de circuit intégré) en silicium, dont l'objet est de faire se recouvrir la surface de ce dernier d'une couche d'oxyde de silicium qui ne se laissera pas traverser par la vapeur dopante au cours d'un processus de dopage par diffusion.

Phosphorus - phosphore. Élément chimique pentavalent que l'on utilise pour le dopage des semiconducteurs. En incorporant du phosphore au silicium, par exemple, on obtient un semiconducteur extrinsèque de type $n$, c'est-à-dire que l'on fait apparaître dans le silicium des porteurs de charges libres qui sont des électrons.

Photoetching - photogravure. Procédé auquel on a recours pour la fabrication des circuits intégrés en vue de reporter, sur la couche protectrice d'oxyde résultant de la passivation, le plan d'un niveau de composants dans la masse du support de silicium ou en surface. Il consiste à ouvrir des fenêtres dans la couche protectrice et se fonde sur la propriété que possèdent les laques photosensibles de se polymériser sous l'action de la lumière (rayonnement ultraviolet).

Photoresist - résine photosensible, laque photosensible. Substance utilisée en photogravure, qui possède la propriété de se polymériser sous l'action de la lumière et, partant, de devenir résistante aux solvants organiques comme le trichloréthylène.

Planetary - nacelle. Dans la technique du dépôt par évaporation sous vide, support recevant la tranche de silicium à la surface de laquelle des composants en couche mince doivent être réalisés.

Polycrystalline - polycristallin. Se dit des substances formées par la juxtaposition d'un très grand nombre de monocristaux (appelés grains cristallins) orientés au hasard. 
Polymerize (to) - se polymériser. Pour un composé monomère de faible masse moléculaire, se transformer, par suite de l'établissement de liaisons entre ses molécules, en un polymère, c'est-àdire en un composé dont la masse moléculaire est un multiple de celle du monomère initial.

Predeposit step - prédiffusion. Première étape de l'opération de dopage par diffusion mise en cuvre pour implanter une zone de conductivité donnée dans la masse d'un support semiconducteur. Elle se déroule dans un four maintenu à une température relativement basse et doit permettre d'obtenir en surface une concentration déterminée de l'impureté choisie comme agent de dopage.

$P$-type semiconductor - semiconducteur de type $\mathbf{p}$. Semiconducteur extrinsèque où la densité des porteurs libres positifs (trous) dépasse celle des électrons de conduction.

Quartz boat - ampoule de quartz, capsule de quartz, nacelle en quartz. Cylindre en verre (quartz) que l'on enfourne dans un four d'oxydation après y avoir placé un lot de tranches de silicium à oxyder en surface.

Resistivity - résistivité. Résistance spécifique caractérisant un corps.

Scribe (to) - rainurer.

Seed - germe, amorce. Petit monoscristal de silicium à partir duquel on fait croître un gros monocristal dénommé lingot et qui est découpé en tranches destinées à servir de supports aux circuits intégrés.

Semiconductor - semiconducteur. Voir intrinsic semiconductor.

Silicon - silicium. Corps pur simple semiconducteur, utilisé sous la forme d'un monocristal comme support de circuit intégré.

Silicon cylinder - lingot de silicium, barreau cylindrique de silicium. Monocristal géant de forme cylindrique, que l'on découpe pour obtenir des tranches destinées à servir de supports à des circuits intégrés monolithiques.

Single crystal - monocristal. Cristal sans interface, dont le réseau conserve en tout point la même orientation.

Slice - Voir wafer.

Slice (to) - découper en tranches.

Solid-state diode - diode solide. Diode constituée par une jonction, c'est-à-dire la juxtaposition d'une zone semiconductrice de type $n$ et d'une zone semiconductrice de type $p$, par opposition à une diode du type lampe à vide.

Thermal diffusion - diffusion thermique. Pénétration relativement lente, sous l'effet de la chaleur, des atomes d'infpureté dans un substrat semiconducteur.

Thin film deposition - dépôt de couches minces.

Ultraviolet radiation - rayonnement ultraviolet. Lumière utilisée en photogravure. Dans la fabrication des circuits intégrés, on s'en sert pour éclairer, à travers un cache, les zones de la couche protectrice d'oxyde du support semiconducteur qui ne doivent pas être mises à nu pour un dopage ou un dépôt.

Unit cell - motif élémentaire. Arrangement des atomes de la maille caractéristique d'un cristal, c'est-à-dire du parallélépipède formé sur les trois vecteurs de base qui expriment la périodicité tridimensionnelle d'un réseau cristallin.

Upset (to) - bouleverser (l'organisation d'un réseau).

Vacuum deposition - dépôt par évaporation sous vide.

Vacuum pump - pompe à vide.

Valence - valence. Mesure de la capacité de combinaison d'un élément chimique. Elle permet de prévoir les affinités chimiques de l'élément et ses modes de liaison avec les autres éléments.

Valence electron - électron de valence. Chacun des électrons des couches externes des atomes qui participent aux liaisons à l'intérieur des édifices atomiques - molécules ou réseaux cristallins.

Vertex - sommet. Point de concours de trois faces d'une maille cristalline.

Window - fenêtre, ouverture. Zone de la surface du support semiconducteur que l'on met à nu en attaquant la couche de passivation (oxyde de silicium) au moyen d'acide après avoir protégé le reste - , de façon à pouvoir réaliser un dopage sélectif.

Wafer - tranche, rondelle, plaquette. Chacun des supports en forme de disque que l'on découpe par sciage dans un lingot de silicium monocristallin. 


\section{BIBLIOGRAPHIE}

GUILLIEN, R. (1969): la Microélectronique, Paris, Presses Universitaires de France, "Que sais-je?", n' 1367, $126 \mathrm{p}$.

HANDEL, S. (1971): A Dictionary of Electronics, Baltimore, Penguin Books, 413 p.

BROSSET, R. et P. FONDANECHE (1969): Dictionnaire mémento d'électronique, Paris, Dunod, $504 \mathrm{p}$

ARNAUD, J.-F. (1972) : Dictionnaire d'électronique, Paris, Larousse, 253 p.

MARKUS, John (1966) : Electronics and Nucleonics Dictionary, New York, McGraw-Hill, $1966,752 \mathrm{p}$.

JACQUES LETHUILLIER 